\title{
Survival Benefit of Traditional Chinese Herbal Medicine (A Herbal Formula for Invigorating Spleen) in Gastric Cancer Patients with Peritoneal Metastasis
}

\author{
Lu Zhao, ${ }^{1,2}$ Ai-Guang Zhao, ${ }^{1}$ Gang Zhao, ${ }^{3}$ Yan Xu, ${ }^{1}$ Xiao-Hong Zhu, ${ }^{1}$ Ni-Da Cao, ${ }^{1}$ \\ Jian Zheng, ${ }^{1}$ Jin-Kun Yang, ${ }^{1}$ and Jian-Hua $\mathrm{Xu}^{2}$ \\ ${ }^{1}$ Department of Oncology, Longhua Hospital Shanghai University of Traditional Chinese Medicine, \\ Shanghai 200032, China \\ ${ }^{2}$ Department of Oncology, Putuo District Central Hospital, Shanghai University of Traditional Chinese Medicine, \\ Shanghai 200062, China \\ ${ }^{3}$ Department of Surgery, Renji Hospital Affiliated to Shanghai Jiao Tong University, Shanghai 200127, China \\ Correspondence should be addressed to Ai-Guang Zhao; 2538312201@qq.com
}

Received 31 October 2013; Revised 11 January 2014; Accepted 25 January 2014; Published 2 March 2014

Academic Editor: Jian-Guo Chen

Copyright (c) 2014 Lu Zhao et al. This is an open access article distributed under the Creative Commons Attribution License, which permits unrestricted use, distribution, and reproduction in any medium, provided the original work is properly cited.

\begin{abstract}
Objective. We evaluated the efficiency of traditional Chinese herbal medicine (a compound herbal formula for invigorating spleen) as a complementary and alternative therapy for gastric cancer patients with peritoneal metastasis. Methods. Between 2001 and 2012, 93 gastric cancer patients with peritoneal metastasis were enrolled in this study. The effect of traditional Chinese herbal medicine on their long-term outcome was investigated. Kaplan-Meier method was used to assess the difference in survival time, and Cox proportional hazards regression analysis was performed to identify independent prognostic factors. Result. First-line palliative chemotherapy plus traditional Chinese herbal medicine was performed in 47 patients and the other 46 patients received chemotherapy alone. The overall survival was different between patients with and without traditional Chinese herbal medicine (12.0 versus 10.5 months; $P=0.046$ ). According to the Cox proportional hazard model, first-line chemotherapy cycle (hazards ratio $[\mathrm{HR}]=0.527 ; 95 \% \mathrm{CI}=0.323 \sim 0.860$ ) and TCHM (hazards ratio $[\mathrm{HR}]=0.644 ; 95 \% \mathrm{CI}=0.481 \sim 0.992$ ) were selected as independent prognostic factors for survival. Conclusion. The results suggest that traditional Chinese herbal medicine could improve the prognosis of the gastric cancer patients with peritoneal metastasis.
\end{abstract}

\section{Introduction}

Peritoneal metastasis is the most common form of recurrence and metastasis in gastric cancer patients. Because peritoneal metastasis has no special manifestation at early stage, once the patients have the serious complications, such as intestinal obstruction, obstructive jaundice, and malignant ascites, they will lead to difficult clinical treatment and poor prognosis. Current therapeutic methods for peritoneal metastasis of gastric cancer include cytoreductive surgery, intraperitoneal chemotherapy, systemic chemotherapy, and biotherapy; but the curative effects are generally unsatisfactory. Therefore, early diagnosis and effective comprehensive treatment is a key target for prolonging the survival time.
Comparatively speaking, traditional Chinese medicine (TCM) is more holistic in treating malignant tumor, whereas Western medicine is more direct and localized through the use of chemotherapy or radiotherapy. The traditional Chinese herbal medicine (TCHM) guided by the concept of holistic not only aims at local treatment, but also focuses on controlling the progression of tumor via adjusting systemic functional status. We designed this TCHM formula (a compound herbal formula for invigorating spleen, formerly named Wei Chang'An) to improve the survival of gastric cancer entirely based on TCM theory.

Our previous researchstudies indicated that this TCHM formula could induce gastric cancer cells apoptosis and suppress proliferation $[1,2]$. Clinical study has indicated that 
this TCHM formula had a potential value in improving the prognosis of patients with advanced gastric cancer [3, 4]. Thus, the aim of the present study was to further investigate the effects of this formula on gastric cancer patients with peritoneal metastasis.

\section{Materials and Methods}

2.1. Patients Characteristics. Between April 2001 and April 2012, 93 gastric cancer patients with peritoneal metastasis who underwent chemotherapy plus TCHM or chemotherapy alone at the Department of Oncology, Longhua Hospital, Shanghai University of Traditional Chinese Medicine, and the Department of Surgery, Renji Hospital, Shanghai Jiao Tong University School of Medicine, were enrolled in this study. The patients' characteristics, pathological findings, and treating processes were collected retrospectively from individual patient records.

2.2. Pretreatment Examinations. The patients were regarded as having clinically evident peritoneal metastasis if they met one of the following clinical or imaging manifestations: (1) intraoperative pathological biopsy proves the dissemination of peritoneum, pouch of Douglas, and nodule, or the discovery of periumbilical metastatic nodules. (2) Positron emission tomography/computed tomography (PET/CT) [5]: $18 \mathrm{~F}-\mathrm{FDG}$ accumulation along peritoneum and intestinal wall and inside omental bursa; SUV $\max \geq 2.5$; the corresponding parts on CT images show morphological changes such as peritoneal thickening and nodules. (3) Magnetic resonance (MR) [6]: abnormally enhanced signals along the surface of mesentery, with unsmooth boundary. (4) Computed tomography (CT) [7]: massive ascites; omental changes (pielike thickening, focal nodules, smudgy density shadow, and bursa-like changes in the omentum); remarkably increased fat density of the small or large bowel.

2.3. Treatment. All patients received first-line palliative chemotherapy depending on their pathological type, physical condition, and tolerance. The scheme was given for 5 to 8 days every 21 to 28 days, based on fluorouracil or together with platinum, anthracycline, or platinum.

The compound herbal formula aimed at invigorating the spleen. The major components were Radix Pseudostellariae (12 g), Rhizoma Atractylodis Macrocephalae (12 g), Poria (30 g), Rhizome Pinelliae Preparata (9 g), green tangerine peel (4.5 g), Concha Ostreae (30 g), and Prunella vulgaris (9 g).

The herbal components above were mainly provided by Sunbow Pharmaceutical (7600 Zhongchun Road, Shanghai 201100, China). This Chinese medicine factory has quality control (certified GMP ShanghaiG0172). The preparation of the formula water decoction and its quality control had been described in our previously published articles [2]. Each dose was $300 \mathrm{~mL}$ at the concentration of $40 \mathrm{~g} / \mathrm{L}$ (Radix Pseudostellariae). One dose per day was taken 2-4 times, and the treatment continued for more than 3 months. According to patients' symptoms and syndrome differentiation, herbs were increased or reduced every 2 weeks.
2.4. Statistical Analysis. Kaplan-Meier method was used to assess the difference in survival time. The differences of the clinical and demographic factors were analyzed by log-rank test. In this study, overall survival (OS) time was defined as time from initial treatment (chemotherapy plus TCHM or chemotherapy alone) to the date of death from any cause.

The Cox proportional hazards regression analysis of survival includes sex, age, primary tumor location, type of histology, number of metastatic sites, first-line chemotherapy cycle, first-line chemotherapy scheme, and intraperitoneal chemotherapy. Differences were assumed to be significant when the $P$ value was less than 0.05 . All of the analyses were performed using the SPSS statistical software program package (SPSS version 16.0 for Windows).

\section{Results}

3.1. Patients. A total of 93 patients were included-47 received the chemotherapy plus TCHM and 46 underwent chemotherapy alone. None of the included patients ever received cytoreductive surgery.

In the patients with TCHM, the longest follow-up period was 111.09 months and the shortest was 3.95 months, 37 patients $(78.7 \%)$ died of cancer, 6 patients $(12.8 \%)$ were alive, and 4 patients (8.5\%) lost followup. And in the patients without TCHM, the numbers were 43.46, 3.25, 44 (95.7\%), $2(4.3 \%)$, and $0(0 \%)$, respectively.

The patients' characteristics and treatments provided are indicated in Table 1. And we compared the baseline between patients according to the treatment provided. There were no differences between any two groups.

3.2. Survival Analysis. Figure 1 shows the overall survival curves of patients with and without TCHM. The median survival time (MST) of patients with TCHM was 12.0 months and that without TCHM was 10.5 months $(P=0.026)$. Overall survival curves of patients who receive $1 \sim 2$ cycles and more than 2 cycles of chemotherapy are shown in Figure 2. MST was significantly longer in patients who receive more than 2 cycles of chemotherapy (13.3 months) than in those who took $1 \sim 2$ cycles (7.0 months, $P=0.002)$.

Multivariate analysis by Cox proportional hazard model showed that first-line chemotherapy cycle $(P=0.010)$ as well as TCHM $(P=0.460)$ were considered as independent prognostic factors for survival. The hazard ratio $(\mathrm{HR}=$ $\operatorname{Exp}[\beta])$ of first-line chemotherapy cycle was 0.527 , and $95 \%$ confidence interval was from 0.323 to 0.860 . The HR of TCHM was 0.644 , and $95 \%$ CI was from 0.418 to 0.992 (Table 2).

\section{Discussion}

Peritoneal metastasis is the predominant pattern among gastric cancer patients [8]. Diagnostic laparoscopy is considered the gold standard for diagnosis of peritoneal metastasis; but as a traumatic test, its clinical application is limited [9]. Peritoneal cytology has been used in the detection of subclinical peritoneal disease, and positive peritoneal cytology 
TABLE 1: Patients and cancer baseline characteristics.

\begin{tabular}{|c|c|c|c|}
\hline \multirow{2}{*}{ Characteristic } & \multicolumn{2}{|c|}{ Treatment } & \multirow{2}{*}{$P$ value } \\
\hline & $\operatorname{TCHM}(n=47)$ & Non-TCHM $(n=46)$ & \\
\hline \multicolumn{4}{|l|}{ Sex (\%) } \\
\hline Male & $26(55.3 \%)$ & $34(73.9 \%)$ & 0.062 \\
\hline \multicolumn{4}{|l|}{ Age (\%) } \\
\hline$<60$ & $29(61.7 \%)$ & $33(71.7 \%)$ & \multirow{2}{*}{0.310} \\
\hline$\geq 60$ & $18(38.3 \%)$ & $13(28.3 \%)$ & \\
\hline \multicolumn{4}{|l|}{ Primary tumor site (\%) } \\
\hline Gastroesophageal junction & $2(4.3 \%)$ & $2(4.3 \%)$ & \multirow{2}{*}{0.983} \\
\hline Stomach & $45(95.7 \%)$ & $44(95.7 \%)$ & \\
\hline \multicolumn{4}{|l|}{ Histology (\%) } \\
\hline Intestinal & $30(63.8 \%)$ & $31(67.4 \%)$ & \multirow{4}{*}{0.973} \\
\hline Diffuse & $3(6.4 \%)$ & $1(2.2 \%)$ & \\
\hline Mixed & $13(27.7 \%)$ & $12(26.1 \%)$ & \\
\hline Missing & $1(2.1 \%)$ & $2(4.3 \%)$ & \\
\hline \multicolumn{4}{|l|}{ Number of metastatic sites (\%) } \\
\hline $1 \sim 2$ & $32(68.1 \%)$ & $31(67.4 \%)$ & \multirow{2}{*}{0.944} \\
\hline$>2$ & $15(31.9 \%)$ & $15(32.6 \%)$ & \\
\hline \multicolumn{4}{|l|}{ Chemotherapy } \\
\hline $1 \sim 2$ cycles & $10(21.3 \%)$ & $16(34.8 \%)$ & \multirow{2}{*}{0.150} \\
\hline 3 cycles and more & $37(78.7 \%)$ & $30(65.2 \%)$ & \\
\hline \multicolumn{4}{|l|}{ First-line chemotherapy scheme } \\
\hline Single-agent regimen ${ }^{\mathrm{a}}$ & $5(10.6 \%)$ & $2(4.3 \%)$ & \multirow{4}{*}{0.945} \\
\hline Joint regimen of 2 drugs $^{\mathrm{b}}$ & $17(36.2 \%)$ & $21(45.7 \%)$ & \\
\hline Joint regimen of 3 drugs $^{c}$ & $21(44.7 \%)$ & $21(45.7 \%)$ & \\
\hline Other regimen $^{\mathrm{d}}$ & $4(8.5 \%)$ & $2(4.3 \%)$ & \\
\hline \multicolumn{4}{|l|}{ Intraperitoneal chemotherapy } \\
\hline Nonintraperitoneal chemotherapy & $40(85.1 \%)$ & $43(93.5 \%)$ & \multirow{2}{*}{0.197} \\
\hline Intraperitoneal chemotherapy & $7(14.9 \%)$ & $3(6.5 \%)$ & \\
\hline
\end{tabular}

${ }^{\mathrm{a}}$ Fluorouracil.

${ }^{\mathrm{b}}$ Fluorouracil plus cisplatin/oxaliplatin.

${ }^{\mathrm{c}}$ Fluorouracil plus cisplatin/oxaliplatin plus anthracycline or paclitaxel/docetaxel.

${ }^{\mathrm{d}}$ Fluorouracil plus others.

was defined as stage IV disease $[10,11]$. PLC, however, has a low sensitivity and is not always positive in cases of visible peritoneal metastasis [12]. Currently, with the development of radiation immunoassay, enzyme-linked immunosorbent assay and RT-PCR for CEA mRNA, its low sensitivity, and high rate of missed diagnosis in detecting the intraperitoneal free cancer cells have been improved $[13,14]$. Meanwhile, PLC is supplemented by the use of imaging techniques such as CT, $\mathrm{MR}$, and PET/CT in the evaluation of peritoneal metastasis. As the diagnosis of peritoneal metastasis remains challenging, in this paper, we evaluated the peritoneal metastasis through focal pathology and imaging manifestations.

So far, there is no standard treatment for peritoneal metastasis. The 4th International Conference on Peritoneal Surface Oncology put forward cytoreductive surgery combined with perioperative intraperitoneal chemotherapy (IPC) for treatment of malignant tumors with peritoneal metastasis [15]. Cytoreductive surgery means that the limited peritoneal metastasis could be locally excised to reduce the tumor load maximally if the patient does not have liver or distant lymph node metastasis and IPC further kills out the micrometastasis and free tumor cells in the abdominal cavity left after surgery. A number of studies have shown that IPC has a positive function on prolonging the survival of advanced gastric cancer patients [16]. Recently, hyperthermia intraperitoneal chemotherapy (HIPEC) is widely applied in clinics, and studies have shown that cytoreductive surgery plus HIPEC were an independent prognostic factor on peritoneal metastasis from GC and recommended it as a standard treatment regimen for selected patients [17-21]. As few cases enrolled in the present study received intraperitoneal treatment (6 in the test group and 4 in the control group), the multifactor analysis did not indicate the IPC as an independent prognostic factor.

As a standard regimen for advanced or recurrent gastric cancer patients from the clinical studies, the efficiency of S1 plus cisplatin for the patients with peritoneal metastasis remains to be seen [22]. Okabe et al. adopted the S-1 combined with cisplatin as induction chemotherapy on 41 
TABLE 2: Multivariate analysis of factors influencing survival of gastric cancer patients with peritoneal metastasis.

\begin{tabular}{lccccccc}
\hline \multirow{2}{*}{ Factor } & \multirow{2}{*}{$B$} & \multirow{2}{*}{$\mathrm{SE}$} & Wald & Sig. & $\operatorname{Exp}(\beta)$ & \multicolumn{2}{c}{$95.0 \% \operatorname{CI}$ for $\operatorname{Exp}(\beta)$} \\
& & & & & Lower & Upper \\
\hline Chemotherapy cycle & -0.640 & 0.250 & 6.570 & 0.010 & 0.527 & 0.323 & 0.860 \\
TCHM & -0.440 & 0.221 & 3.977 & 0.046 & 0.644 & 0.418 & 0.992 \\
\hline
\end{tabular}

gastric cancer patients with peritoneal metastasis. The result showed that, after chemotherapy, the survival benefit was significant in the patients with R0 resection compared to those with noncurative resection or without surgery, and it purposed that gastric cancer patients with limited peritoneal dissemination were sensitive to induction chemotherapy with S-1 plus cisplatin [23]. Due to its good transition from blood to the peritoneal cavity, paclitaxel was considered to be effective for peritoneal metastasis [24, 25]. In the OGSG0401 trail, the median survival time of gastric cancer patients with peritoneal metastasis who received treatment of paclitaxel (50 mg/m $\mathrm{m}^{2}$ qd day 1 and 8 ) plus S-1 (40 mg/m $\mathrm{m}^{2}$ bid day $\left.1-14\right)$ scheme was 310 days, and the regimen demonstrated efficacy and tolerable toxicity [26]. The gastric cancer patients with peritoneal metastasis were excluded from clinical trials containing cisplatin for their complications such as ascites. MTX and 5-Fu sequential scheme was used based on the result from Japan Clinical Oncology Group (JCOG) 0106 trial for such patients [27]. Imazawa et al. reported that after treatment of methotrexate (MTX) combined with 5-Fu chemotherapy, the 31 gastric cancer patients with peritoneal metastasis had median OS of 255 days; of the 21 detectable focus patients, PR was observed in 4 cases; of the 26 patients complicated with ascites, the ascites amount decreased obviously in 14 cases [28]. Since effective regimen is under discussion to improve the poor prognosis of peritoneal metastasis from gastric cancer, systemic chemotherapy is the main treatment. Studies above showed that chemotherapy could improve the overall survival of gastric cancer patients with peritoneal metastasis. In our study, Cox proportional hazard model also showed that the chemotherapeutic cycle was an independent influence factor of prognosis. Nevertheless, the chemotherapy scheme was not indicated as an influence factor.

TCM developed from a totally different background from Western medicine. We designed this formula to improve the survival of gastric cancer based on TCM theory of the etiology and pathogenesis. In this compound formula, the herbs which have the function of invigorating the spleen function played the role of the sovereign ingredient. And the herbs for heat-clearing and detoxifying as well as for promoting circulation and removing blood stasis played the roles of minister and assistant ingredients. The major ingredients in this formula were Radix Pseudostellariae, Rhizoma Atractylodis Macrocephalae, Poria, Rhizome Pinelliae Preparata, green tangerine peel, Concha Ostreae, and Prunella vulgaris. And the attributes of the constituents of this compound formula are as follows: Radix Pseudostellariae and Rhizoma Atractylodis Macrocephalae are used to reinforce the spleen function supplement Qi (according to the fundamental theory of TCM, Qi is often translated as vital energy);
Poria is used to invigorate the spleen and eliminate dampness; Rhizome Pinelliae Preparata is used to dry dampness and dissolve phlegm; green tangerine peel is used to promote the circulation of Qi and blood; Concha Ostreae and Prunella vulgari are used to soften hardness and dissolve lump.

In our previous studies, it was found that the TCHM (a herbal formula for invigorating the spleen) had certain antiperitoneal metastasis ability, a significant reduction in peritoneal metastasis lesions and ascites occurrence in the model of nude mice which were planted splenicsubcapsularly with human gastric cancer cell strain SGC7901 [29]. And the TCHM could induce gastric cancer cell apoptosis and inhibit growth in vivo. Its mechanisms might be involved in the downregulation of Stat3, RIPX, ROD1, and Bcl-2 genes $[1,2]$.

Previous clinical paired comparative studies have indicated that advanced gastric cancer patients could benefit from TCHM. A clinical study of 399 patients with advanced gastric cancer suggested that patients who had received TCHM had better prognosis on multivariate analysis, independent of other prognostic factors $(P=0.000)$ [3]. In this present study, the OS of advanced gastric cancer with peritoneal metastasis could be prolonged by TCHM (12.0 versus 10.5 months; $P=$ 0.046). Meanwhile, the multifactor analysis also showed that the TCHM has an independent prognostic factor $(P=0.460)$. The hazard ratio of TCHM was 0.644 , and $95 \%$ CI was from 0.418 to 0.992 .

Though there is limitation associated with retrospective study in providing robust evidence, the result of this study revealed the positive curative effectiveness of TCHM as a combination therapy for gastric cancer patients with peritoneal metastasis.

Based on our result, we proposed that TCHM aiming at invigorating the spleen combined with systematic chemotherapy could be useful for the treatment of gastric cancer with peritoneal metastasis. And with the development of cytoreductive surgery and intraperitoneal chemotherapy, there is still much room for the limited survival rates to improve.

Safety and Toxicity. The TCHM treatment was well tolerated. During the followup, there were no TCHM-related adverse events observed.

\section{Conclusions}

In this study, we showed that the traditional Chinese herbal medicine in combination with chemotherapy would improve the prognosis of gastric cancer patients with peritoneal metastasis than chemotherapy alone. 


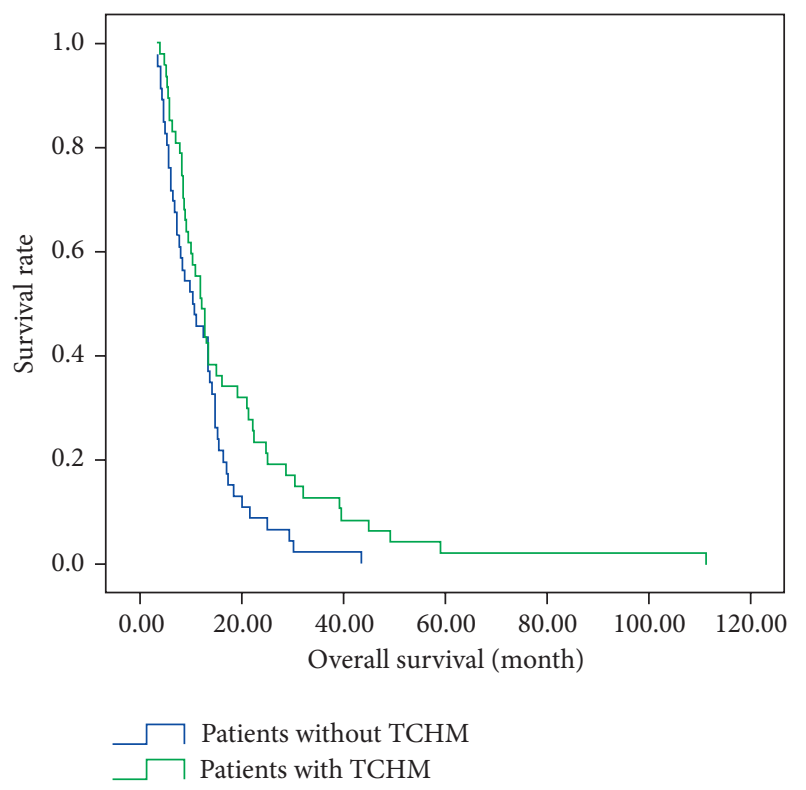

FIGURE 1: Survival curves of patients with and without TCHM. There is difference in MST between patients with TCHM (12.0 months) and those without TCHM (10.5 months; $P=0.026)$.

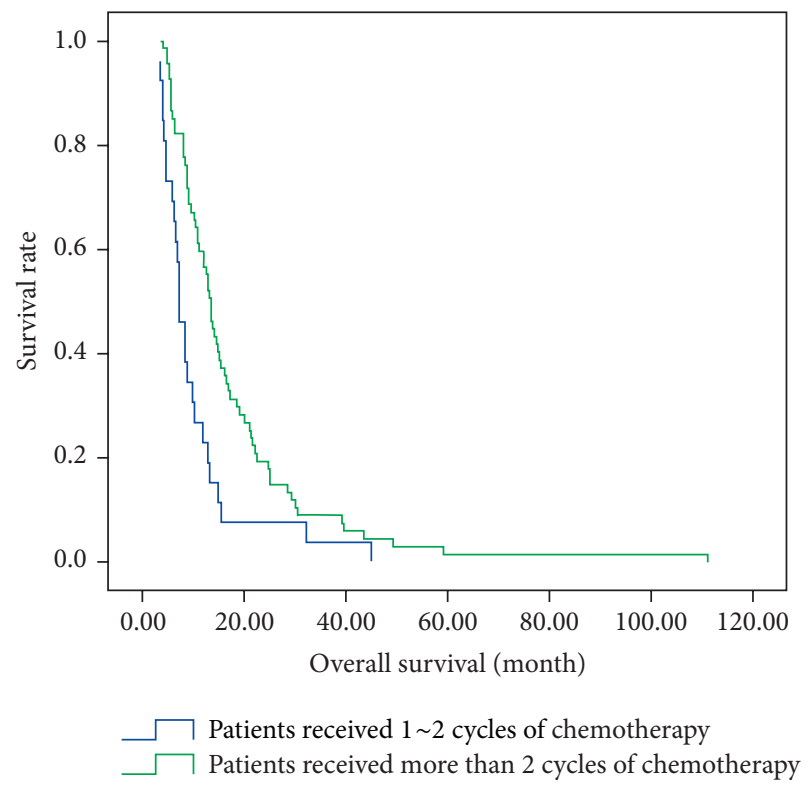

FIGURE 2: Survival curves of patients who receive 1 2 cycles and more than 2 cycles of chemotherapy. MST was significantly longer for patients who receive more than 2 cycles of chemotherapy (13.3 months) than those who received $1 \sim 2$ cycles (7.0 months; $P=0.002$ ).

\section{Conflict of Interests}

The authors declared that there is no potential conflict of interests.

\section{Funding}

This study was funded by the National Natural Science Foundation of China (81373861) and Science and Technology
Commission of Shanghai Municipality (Science and Technology Innovation Plan, 13401903700).

\section{Acknowledgments}

The authors are grateful to all the participating patients and their families to complete this study.

\section{References}

[1] A. G. Zhao, H. L. Zhao, X. J. Jin, J. K. Yang, and L. D. Tang, "Effects of Chinese Jianpi herbs on cell apoptosis and related gene expression in human gastric cancer grafted onto nude mice," World Journal of Gastroenterology, vol. 8, no. 5, pp. 792796, 2002.

[2] A. G. Zhao, T. Li, S. F. You et al., "Effects of Wei Chang An on expression of multiple genes in human gastric cancer grafted onto nude mice," World Journal of Gastroenterology, vol. 14, no. 5, pp. 693-700, 2008.

[3] Y. Xu, A. G. Zhao, Z. Y. Li et al., "Survival benefit of traditional Chinese herb medicine (a herbal formual for invigorating spleen) for patients with advanced gastric cancer," Integrative Cancer Therapies, vol. 12, no. 5, pp. 414-422, 2013.

[4] A. G. Zhao, Y. Cai, J. K. Yang, J. Zheng, and K. P. Shen, "Effect of Chinese Jianpi herbs on prognosis of gastric cancer," World Chinese Journal of Digestology, vol. 13, no. 9, pp. 1055-1058, 2005.

[5] Z. Wang and J. Q. Chen, "Imaging in assessing hepatic and peritoneal metastases of gastric cancer: a systematic review," BMC Gastroenterology, vol. 11, article 19, 2011.

[6] M. Bozkurt, S. Doganay, M. Kantarci et al., "Comparison of peritoneal tumor imaging using conventional MR imaging and diffusion-weighted MR imaging with different b values," European Journal of Radiology, vol. 80, no. 2, pp. 224-228, 2011.

[7] J. E. Kim, M. W. Lee, J. W. Moon et al., "CT detection of dry peritoneal seeding in patients with advanced gastric cancer," Hepatogastroenterology, vol. 59, no. 119, pp. 2202-2206, 2012.

[8] K. Maruyama, M. Kaminishi, K. I. Hayashi et al., "Gastric cancer treated in 1991 in Japan: data analysis of nationwide registry," Gastric Cancer, vol. 9, no. 2, pp. 51-66, 2006.

[9] P. J. Karanicolas, E. B. Elkin, L. M. Jacks et al., "Staging laparoscopy in the management of gastric cancer: a populationbased analysis," Journal of the American College of Surgeons, vol. 213, no. 5, pp. 644-e1, 2011.

[10] D. Bentrem, A. Wilton, M. Mazumdar, M. Brennan, and D. Coit, "The value of peritoneal cytology as a preoperative predictor in patients with gastric carcinoma undergoing a curative resection," Annals of Surgical Oncology, vol. 12, no. 5, pp. 347-353, 2005.

[11] S. B. Edge, D. R. Byrd, C. C. Compton, A. G. Fritz, F. L. Greene, and A. Trotti, American Joint Committee on Cancer (AJCC) Cancer Staging Manual, Springer, Chicago, Ill, USA, 7th edition, 2010.

[12] M. B. Wilkiemeyer, S. C. Bieligk, R. Ashfaq, D. B. Jones, R. V. Rege, and J. B. Fleming, "Laparoscopy alone is superior to peritoneal cytology in staging gastric and esophageal carcinoma," Surgical Endoscopy and Other Interventional Techniques, vol. 18, no. 5, pp. 852-856, 2004.

[13] M. La Torre, M. Ferri, M. R. Giovagnoli et al., "Peritoneal wash cytology in gastric carcinoma. Prognostic significance and therapeutic consequences," European Journal of Surgical Oncology, vol. 36, no. 10, pp. 982-986, 2010. 
[14] J. Wong, K. J. Kelly, A. Mittra et al., "RT-PCR increases detection of submicroscopic peritoneal metastases in gastric cancer and has prognostic significance," Journal of Gastrointestinal Surgery, vol. 16, pp. 889-896, 2012.

[15] S. González-Moreno, "Peritoneal surface oncology: a progress report," European Journal of Surgical Oncology, vol. 32, no. 6, pp. 593-596, 2006.

[16] T. D. Yan, D. Black, P. H. Sugarbaker et al., "A systematic review and meta-analysis of the randomized controlled trials on adjuvant intraperitoneal chemotherapy for resectable gastric cancer," Annals of Surgical Oncology, vol. 14, no. 10, pp. 27022713, 2007.

[17] O. Glehen, V. Schreiber, E. Cotte et al., "Cytoreductive surgery and intraperitoneal chemohyperthermia for peritoneal carcinomatosis arising from gastric cancer," Archives of Surgery, vol. 139, no. 1, pp. 20-26, 2004.

[18] S. Scaringi, R. Kianmanesh, J. M. Sabate et al., "Advanced gastric cancer with or without peritoneal carcinomatosis treated with hyperthermic intraperitoneal chemotherapy: a single western center experience," European Journal of Surgical Oncology, vol. 34, no. 11, pp. 1246-1252, 2008.

[19] X. J. Yang, C. Q. Huang, T. Suo et al., "Cytoreductive surgery and hyperthermic intraperitoneal chemotherapy improves survival of patients with peritoneal carcinomatosis from gastric cancer: final results of a phase III randomized clinical trial," Annals of Surgical Oncology, vol. 18, no. 6, pp. 1575-1581, 2011.

[20] B. Goslin, S. Sevak, A. Siripong et al., "Outcomes of cytoreduction with hyperthermic intraperitoneal chemotherapy: our experience at a midwest community hospital," American Journal of Surgery, vol. 203, no. 3, pp. 383-387, 2012.

[21] W. K. Bae, M. S. Park, J. H. Lee et al., "Docetaxel-loaded thermoresponsive conjugated linoleic acid-incorporated poloxamer hydrogel for the suppression of peritoneal metastasis of gastric cancer," Biomaterials, vol. 34, no. 4, pp. 1433-1441, 2013.

[22] T. Sano and T. Aiko, "New Japanese classifications and treatment guidelines for gastric cancer: revision concepts and major revised points," Gastric Cancer, vol. 14, no. 2, pp. 97-100, 2011.

[23] H. Okabe, S. Ueda, K. Obama, H. Hosogi, and Y. Sakai, "Induction chemotherapy with S-1 plus cisplatin followed by surgery for treatment of gastric cancer with peritoneal dissemination," Annals of Surgical Oncology, vol. 16, no. 12, pp. 3227-3236, 2009.

[24] J. Sakamoto, T. Matsui, and Y. Kodera, "Paclitaxel chemotherapy for the treatment of gastric cancer," Gastric Cancer, vol. 12, no. 2, pp. 69-78, 2009.

[25] S. Iwasa, M. Goto, H. Yasui et al., "Multicenter feasibility study of combination therapy with fluorouracil, leucovorin and paclitaxel (FLTAX) for peritoneal disseminated gastric cancer with massive ascites or inadequate oral intake," Japanese Journal of Clinical Oncology, vol. 42, no. 9, pp. 787-793, 2012.

[26] Y. Kimura, H. Machida, K. Fujitani et al., "Combination of S1 and paclitaxel for advanced/recurrent gastric cancer patients with peritoneal metastasis, feasibility study (OGSG0401)," Gan to Kagaku Ryoho, vol. 37, no. 1, pp. 151-155, 2010.

[27] K. Shirao, N. Boku, Y. Yamada et al., "Randomized phase III study of 5-fluorouracil continuous infusion (5FUci) versus methotrexate and 5-FU sequential therapy (MF) in gastric cancer with peritoneal metastasis (JCOG0106)," Journal of Clinical Oncology, vol. 27, no. 15S, abstract 4545, 2009.

[28] M. Imazawa, T. Kojima, N. Boku et al., "Efficacy of sequential methotrexate and 5-fluorouracil (MTX/5FU) in improving oral intake in patients with advanced gastric cancer with severe peritoneal dissemination," Gastric Cancer, vol.12, no. 3, pp. $153-$ $157,2009$.

[29] H. L. Zhao, A. G. Zhao, S. F. You, Y. Gu, L. D. Tang, and J. K. Yang, "Growth-inhibiting and anti-metastasis effects of Weichangan Decoction on orthotopic transplant nude mouse model of human gastric cancer," Journal of Chinese Integrative Medicine, vol. 3, no. 5, pp. 378-381, 2005. 


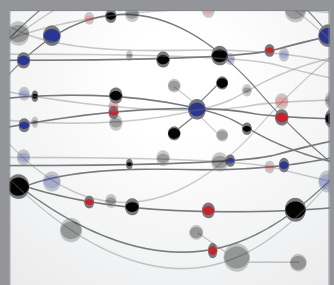

The Scientific World Journal
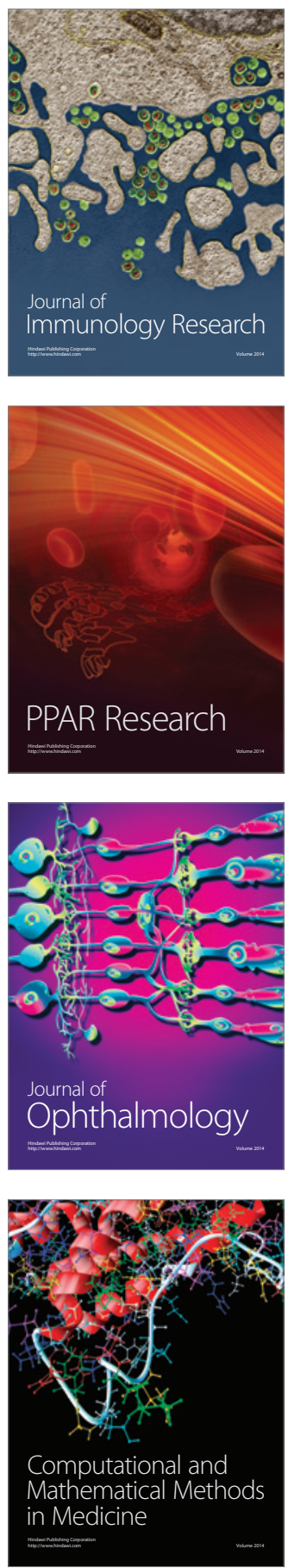

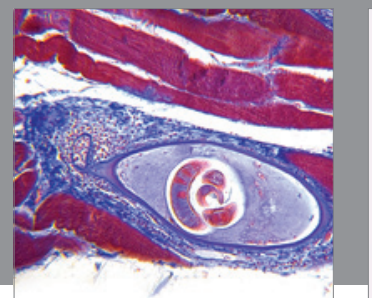

Gastroenterology

Research and Practice
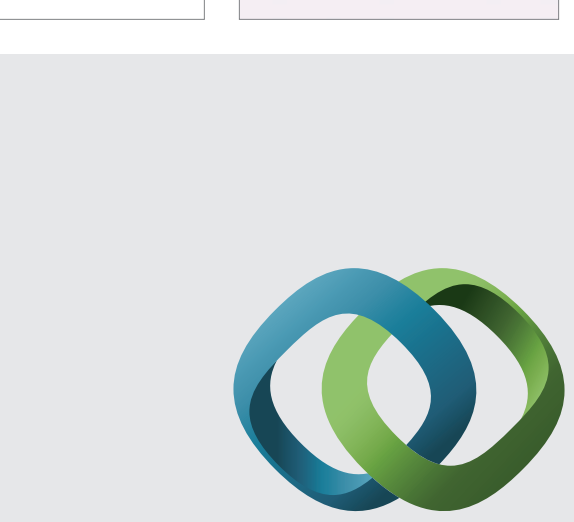

\section{Hindawi}

Submit your manuscripts at

http://www.hindawi.com
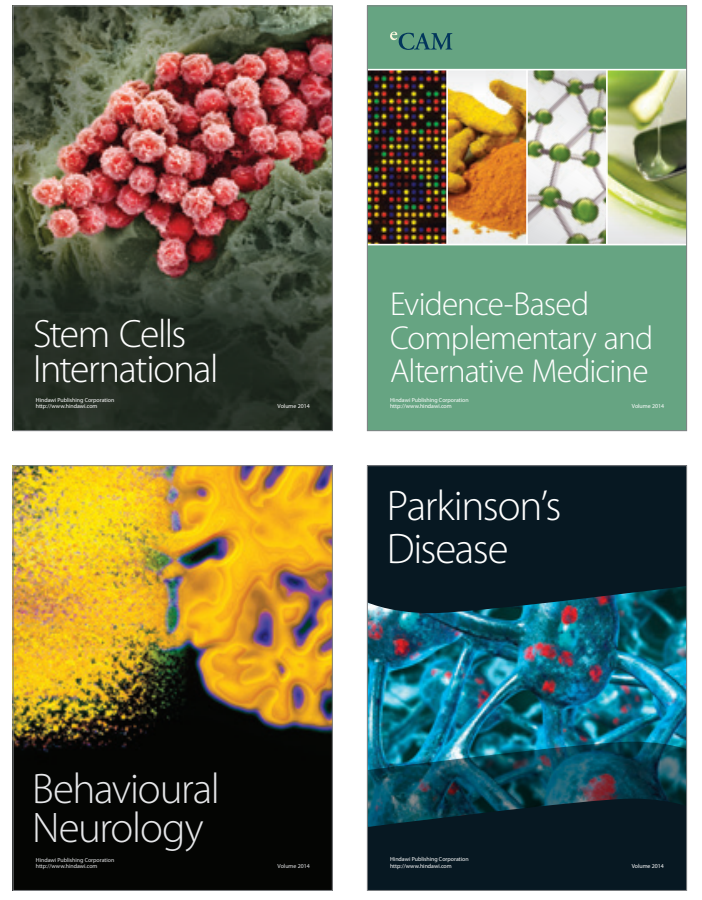
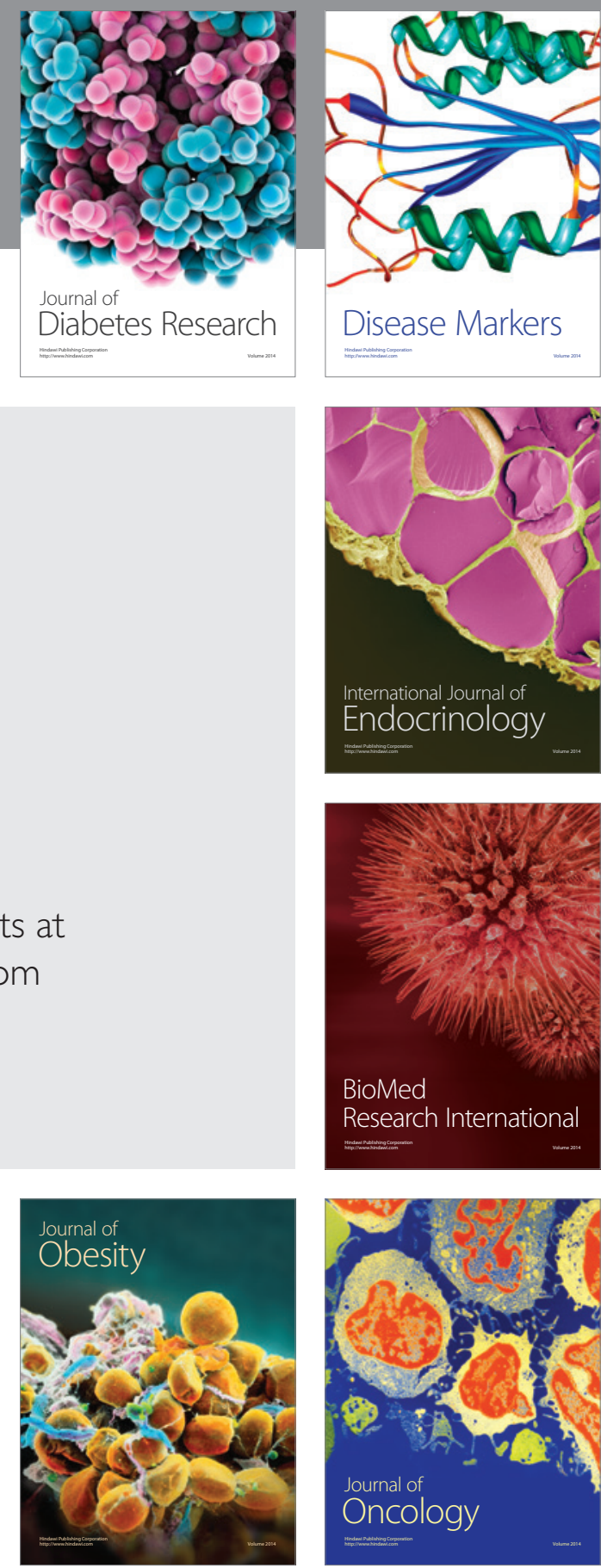

Disease Markers
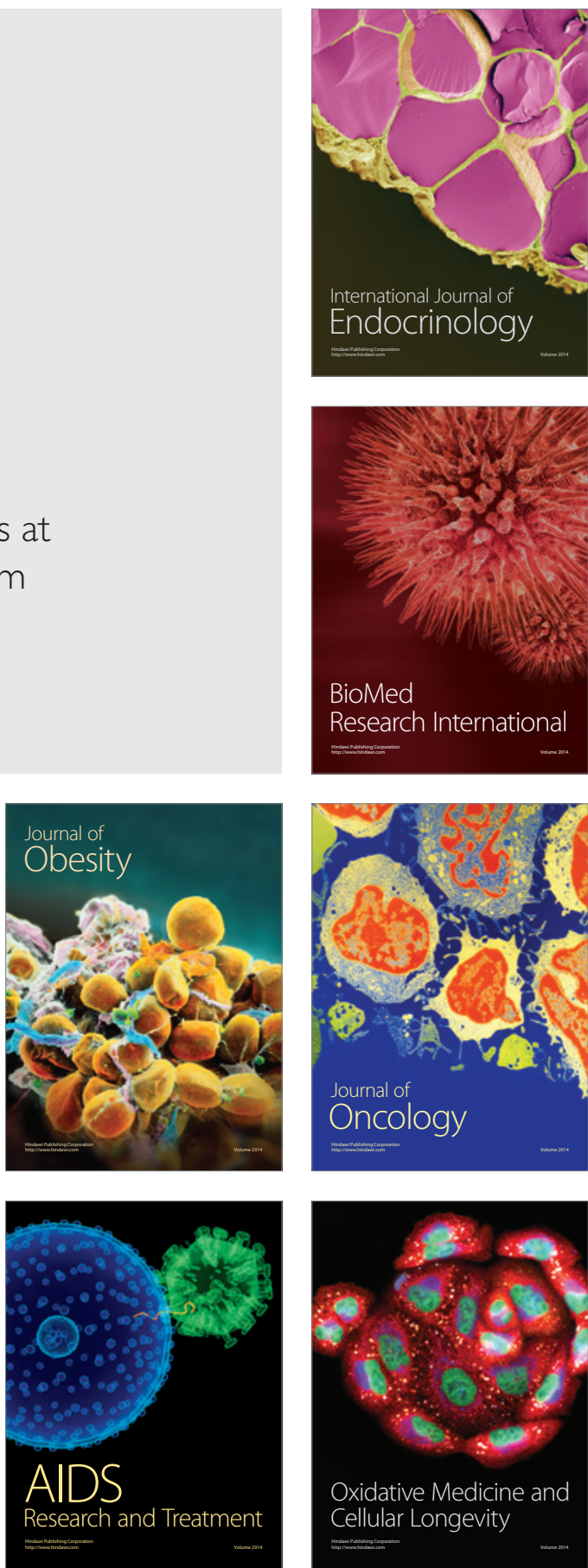